\title{
Applications of molecular neuro-oncology - a review of diffuse glioma integrated diagnosis and emerging molecular entities
}

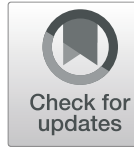

\author{
Matthew D. Wood ${ }^{1 *}$ (D, Aaron M. Halfpenny ${ }^{1}$ and Stephen R. Moore ${ }^{2}$
}

\begin{abstract}
Insights into the molecular underpinnings of primary central nervous system tumors have radically changed the approach to tumor diagnosis and classification. Diagnostic emphasis has shifted from the morphology of a tumor under the microscope to an integrated approach based on morphologic and molecular features, including gene mutations, chromosomal copy number alterations, and gene rearrangements. In 2016, the World Health Organization provided guidelines for making an integrated diagnosis that incorporates both morphologic and molecular features in a subset of brain tumors. The integrated diagnosis now applies to infiltrating gliomas, a category that includes diffusely infiltrating astrocytoma grades II, III, and IV, and oligodendroglioma, grades II and III, thereby encompassing the most common primary intra-axial central nervous system tumors. Other neoplasms such as medulloblastoma, embryonal tumor with multilayered rosettes, certain supratentorial ependymomas, and atypical teratoid/rhabdoid tumor are also eligible for integrated diagnosis, which can sometimes be aided by characteristic immunohistochemical markers. Since 2016, advances in molecular neuro-oncology have resulted in periodic updates and clarifications to the integrated diagnostic approach. These advances reflect expanding knowledge on the molecular pathology of brain tumors, but raise a challenge in rapidly incorporating new molecular findings into diagnostic practice. This review provides a background on the molecular characteristics of primary brain tumors, emphasizing the molecular basis for classification of infiltrating gliomas, the most common entities that are eligible for an integrated diagnosis. We then discuss entities within the diffuse gliomas that do not receive an integrated diagnosis by WHO 2016 criteria, but have distinctive molecular features that are important to recognize because their clinical behavior can influence clinical management and prognosis. Particular attention is given to the histone H3 G34R/G34V mutant astrocytomas, an entity to consider when faced with an infiltrating glioma in the cerebral hemisphere of children and young adults, and to the group of histologically lower grade diffuse astrocytic gliomas with molecular features of glioblastoma, an important category of tumors to recognize due to their aggressive clinical behavior.
\end{abstract}

Keywords: Brain tumors, Molecular pathology, Diffuse glioma, Integrated diagnosis, WHO 2016

\section{Background}

Primary brain tumors encompass many distinct tumor types arising in the brain parenchyma or meninges, with varying prevalence based on patient age and tumor location. The most common malignant primary brain tumors are gliomas, a category of tumors arising from glial or glial precursor cells that includes astrocytomas (which

\footnotetext{
* Correspondence: woodmat@OHSU.edu

'OHSU Department of Pathology, Division of Anatomic Pathology, Section of Neuropathology, Oregon Health \& Science University, 3181 SW Sam Jackson Park Road, L-113, Portland, OR 97213, USA

Full list of author information is available at the end of the article
}

may be diffuse or circumscribed), oligodendrogliomas, ependymomas, and other rare histologic groups. Together, gliomas account for about $75 \%$ of malignant primary brain tumors, and the vast majority are glioblastoma [1]. This review focuses on the molecular features of diffuse gliomas, a category of tumors defined by infiltrating neoplastic cells invading through the brain or spinal cord parenchyma without a distinct margin. Notably, advances in molecular characterization of brain tumors have occurred well beyond this histologic category. Other tumor categories with important molecular pathologic features include the circumscribed 
astrocytomas such as pilocytic astrocytoma, pleomorphic xanthoastrocytoma, and subependymal giant cell astrocytoma, all of which are more frequent in children and young adults. Embryonal tumors are a category of aggressive, poorly-differentiated tumors, also more common in children, and mostly accounted for by medulloblastomas [2]. Other embryonal tumors such as atypical teratoid/rhabdoid tumor (ATRT) and embryonal tumor with multilayered rosettes (ETMR) are rare, but important to recognize due to their aggressive behavior and distinct molecular underpinnings. Glioneuronal tumors include tumors with a mixture of glial and neuronal differentiation, and as a group they are mostly low grade. Glioneuronal tumors show significant histologic and molecular overlap, which can make the diagnosis challenging $[3,4]$. References cited in this article provide details on many of these other entities, and their molecular features.

The article will address the histologic categories of diffuse astrocytoma, anaplastic astrocytoma, glioblastoma, oligodendroglioma, anaplastic oligodendroglioma, and the key histologic and molecular features seen with these morphologies. The diffuse gliomas require molecular information for classification, and these tumors are regularly encountered in routine practice. Understanding the relationship between diffuse glioma histologic and molecular features is critical for recognizing cases that require molecular studies. The morphologic basis for glioma classification was established in 1926 by Bailey and Cushing, who devised a set of histologic categories and introduced a naming convention based on tumor resemblance to normal cellular counterparts in the developing nervous system [5-7]. This work set the foundation for morphologic classification of gliomas, which was the mainstay of classification for almost a century. Brain tumor classification was eventually codified in the 1979 first edition of the World Health Organization (WHO) guidelines for central nervous system (CNS) tumor classification, and subsequent editions were published in 1993, 2000, and the 4th edition in 2007.

In recent decades, molecular studies of brain tumors resulted in an exponential rise in our knowledge of the molecular underpinnings of these neoplasms (Fig. 1). From the 1998 identification of chromosome arm 1p and $19 \mathrm{q}$ loss as a favorable prognostic indicator in infiltrating gliomas and the recognition of the association with oligodendroglioma histology, to the 2008 identification of isocitrate dehydrogenase mutations as an early driver of gliomagenesis, molecular advances have provided critical information that now has implications for brain tumor treatment and prognosis [8-10]. For the infiltrating gliomas, molecular characterization culminated in 2015 with two large studies showing that molecular

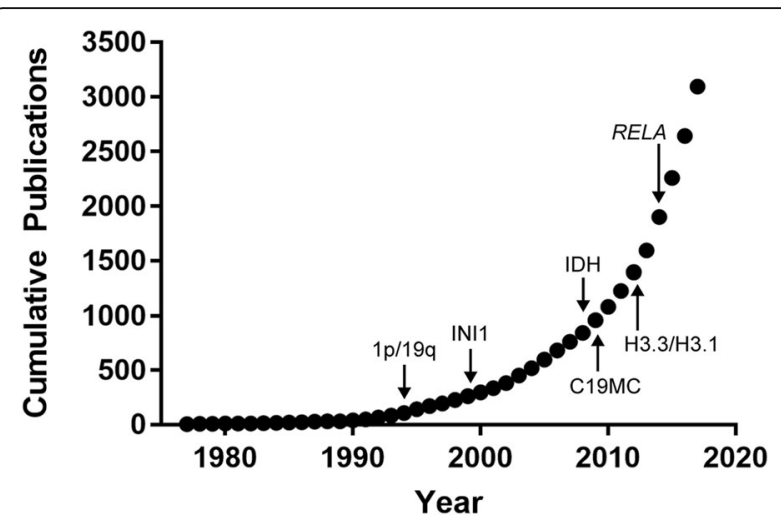

Fig. 1 Exponential growth in brain tumor molecular information. Pubmed search results for the combined terms "brain tumor" and "molecular" from 1979 to 2017. Annotations show the year of identification for key molecular features that were incorporated into the WHO 2016 diagnostic recommendations, including 1p/19qcodeletion for oligodendroglioma (1p/19q), loss of INI1 protein for atypical teratoid/rhabdoid tumor (INI1), isocitrate dehydrogenase alterations for lower grade infiltrating gliomas (IDH), histone $\mathrm{H3}$ mutations for diffuse midline and other pediatric high-grade gliomas (H3.3/H3.1), RELA fusions for supratentorial ependymoma (RELA), and chromosome 19 microRNA cluster alterations for embryonal tumor with multilayered rosettes (C19MC)

classification of these tumors more reliably reflected underlying tumor biology than traditional morphology $[11,12]$. When lower-grade diffuse gliomas were grouped in an unbiased manner by molecular profiling, key molecular alterations like isocitrate dehydrogenase mutation and chromosome arms $1 \mathrm{p}$ and $19 \mathrm{q}$ codeletion distinguished the molecular subgroups, but traditional histopathologic features - especially mixed "oligoastrocytoma" morphology - were seen in multiple molecular subgroups [12, 13]. Subsequently, in 2016 a revised 4th edition of the WHO Classification of Tumours of the Central Nervous System (WHO 2016) officially incorporated molecular features into CNS tumor classification $[14,15]$. The WHO 2016 added new diagnostic entities, removed others, and updated recommendations on histologic assessment of certain tumors. Many excellent review articles have discussed these revisions, either in general or focusing on gliomas, pediatric tumors, clinical applications, practical approaches to molecular diagnostic testing, and more [14, 16-21].

The aim of this review is to provide an overview of the molecular underpinning of the infiltrating gliomas, the most common brain tumors that should receive an integrated molecular diagnosis by WHO 2016 criteria. Various testing methods exist for obtaining molecular data, and some advantages, limitations, and potential pitfalls of the more common approaches will be discussed. Building on this foundation, we will discuss some molecular entities within infiltrating gliomas that should be considered when a pathologist encounters an unusual 
tumor that does not seem to fit into an existing molecular category. Molecular pathology of brain tumors as a general topic applies to many other glial and non-glial neoplasms which are not discussed in this review. Readers are referred to several recent focused reviews which have addressed other areas of the molecular pathology of other brain tumors, such as glioneuronal tumors, meningothelial tumors, other mesenchymal tumors, tumors of the sellar region, and lymphomas and histiocytic tumors [20-23].

\section{The ISN-Haarlem guidelines for reporting an integrated diagnosis}

In 2014, the International Society of Neuropathologists (ISN) convened an expert group of over two dozen neuropathologists in Haarlem, the Netherlands, to address challenges in standardization of reporting integrated diagnoses. This group incorporated their expertise with input from over 150 neuro-oncology specialists to set boundaries and priorities for molecular testing in brain tumors. The group prioritized defining entities as narrowly as possible in order to create homogeneous tumor groups, and created a four-layered system to standardize reporting of integrated tumor classification [24]. The ISN-Haarlem system allows separation of the molecular information about a tumor (layer 4), the WHO grade (layer 3), and the histologic classification (layer 2) from the final integrated diagnosis which includes molecular and morphologic characteristics (layer 1), providing more granular information than a single integrated diagnosis alone. This streamlines reporting for cases where the histologic classification appears to conflict with the tumor grade, such as in diffuse midline glioma with histone H3 K27M-mutation, which is considered WHO grade IV based on molecular findings, even with lower-grade histology.

Molecular testing will not be possible in all cases, as tissue quality and quantity are frequent limiting factors in brain tumor sampling and testing may subject to technical failure. The WHO does not endorse specific testing modalities, and each institution can choose from several platforms or approaches [15]. Still, some centers may not have access to the necessary testing to support an integrated diagnosis. The WHO classification still allows reporting of a histologic diagnosis followed by the qualifier "not otherwise specified" (NOS) to reflect that complete molecular information is not available, or testing cannot be performed $[15,25]$. In cases where molecular information is obtained, but the results do not fit into an existing diagnostic category, a different qualifier of "not elsewhere classified" (NEC) may be applied [25]. Such cases are presumed to be provisional, and this category should be used with decreasing frequency over time, with expanding molecular studies of brain tumors.

\section{CIMPACT-NOW: a vehicle for updates to CNS tumor molecular diagnostics}

The Consortium to Inform Molecular and Practical Approaches to CNS Tumor Taxonomy (cIMPACT-NOW) was established to create a mechanism for updates in between WHO editions, which have historically been separated by intervals of at least 7 years [26, 27]. The consortium created working committees to address specific questions about molecular diagnosis. The consortium has released guidelines on topics such as how to use the NOS and NEC qualifiers in diagnostic reports, requirements for $1 \mathrm{p} / 19 \mathrm{q}$ testing in infiltrating gliomas with astrocytic morphology, and the criteria for the diagnosis of diffuse midline glioma, H3 K27M-mutant, WHO grade IV $[25,28]$. A critical and very recent update concerns the molecular criteria that indicate aggressive behavior in IDH-wildtype diffuse or anaplastic astrocytoma, which is discussed later in this review [29]. Updates from cIMPACT-NOW are to be reported in the journal Acta Neuropathologica with an accompanying editorial in Brain Pathology, and both will be a valuable resource for keeping up to date on molecular pathology of brain tumors.

\section{Histologic classification and grading of diffuse gliomas}

Diffusely infiltrating gliomas are defined by a growth pattern of individual tumor cells growing through the brain parenchyma, in contrast with the sharp, pushing border seen in circumscribed gliomas or metastatic tumors [30]. Individual tumor cells may be seen surrounding entrapped neurons, clustering around small vessels, and accumulating in the subpial space. These "secondary structures" are highly specific for diffuse glioma. Conventional astrocytic histologic features are elongated, hyperchromatic tumor cell nuclei with irregular nuclear contours and scant associated cytoplasm (Fig. 2, a-c). Oligodendroglial features include uniform, round to oval nuclei with crisp nuclear borders, delicate speckled chromatin, and (in formalin fixed tissue) perinuclear cytoplasmic clearing, with a background of delicate branching small vessels (Fig. 2, d-e) [31]. Diffuse glioma grading is based on mitotic activity, vascular proliferation, and necrosis. Diffuse astrocytomas with significant mitotic activity are considered WHO grade III (anaplastic) while glioblastoma, WHO grade IV, is defined by the presence of vascular proliferation and/or necrosis, the latter feature being often (but not always) palisading (Fig. 2, b-c). Conventional oligodendroglioma is WHO grade II, while findings of high cellularity, cytologic atypia, necrosis, vascular proliferation, and significant mitotic activity are features for designating anaplastic oligodendroglioma, WHO grade III, with a minimum of "conspicuous microvascular proliferation and/or brisk mitotic activity" required for the diagnosis (Fig. 2, d-e) 


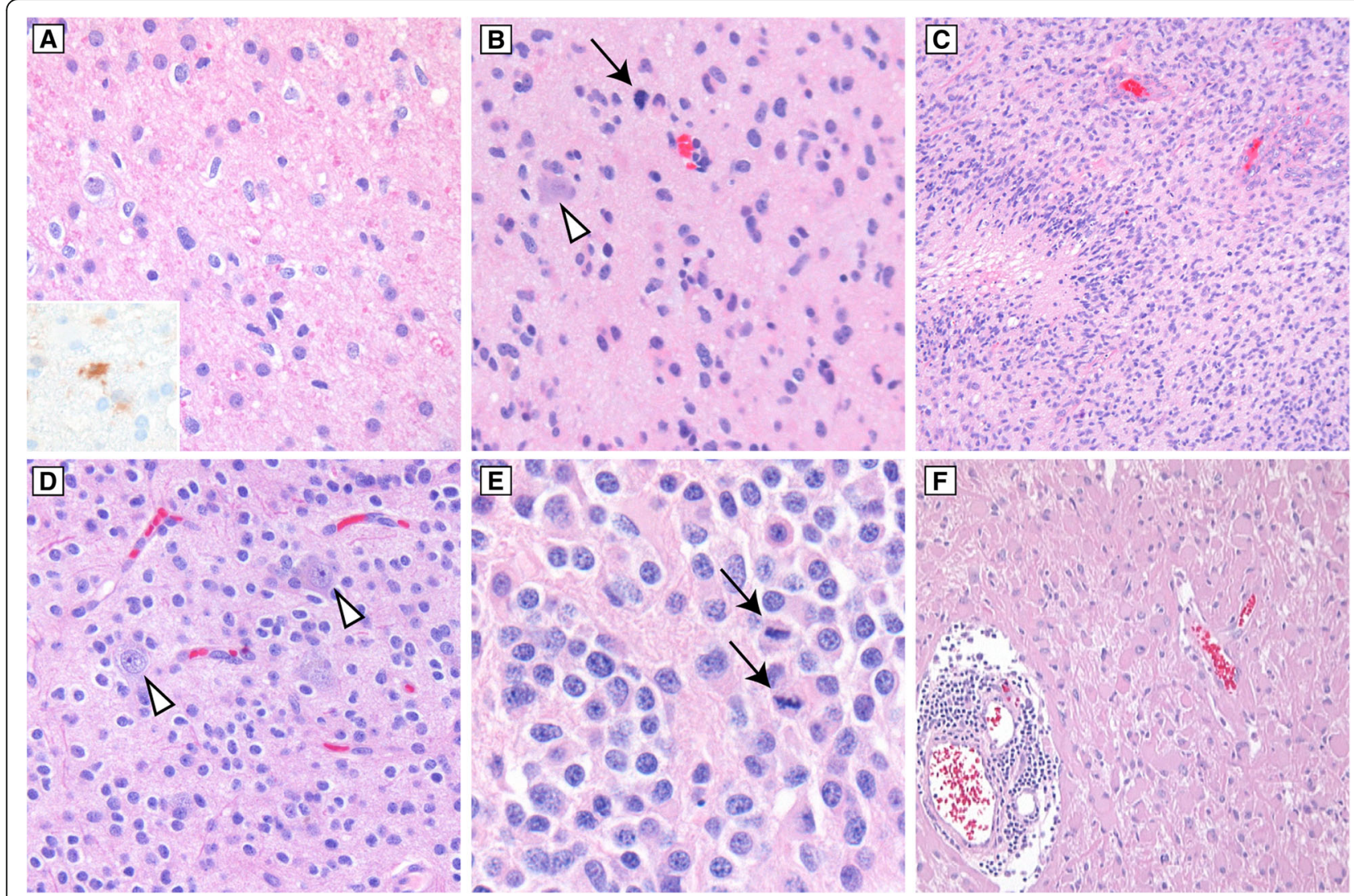

Fig. 2 Typical histologic features of infiltrating gliomas. In diffuse astrocytomas (a), cellularity is increased due to infiltrating neoplastic astrocytes with irregular, hyperchromatic nuclei and scant associated cytoplasm. Immunohistochemistry for IDH1 R132H mutant protein (A, inset) can be helpful when infiltrating cells are sparse or rare. $\mathbf{b}$ Anaplastic astrocytoma is distinguished by mitotic activity (black arrow). Note the infiltrating tumor cells around a non-neoplastic neuron (white arrowhead). c Palisading necrosis (left) and endothelial proliferation (upper right) are histologic features of glioblastoma, though neither feature is absolutely specific. $\mathbf{d}$ WHO grade II oligodendroglioma with uniform, rounded nuclei and perinuclear clearing, the latter feature being an artifact of formalin fixation. Infiltrative growth is demonstrated by entrapped non-neoplastic neurons (white arrowheads). e Oligodendrogliomas with high cellularity, cytologic atypia, significant mitotic activity (black arrows) generally defined as 6 or more mitoses per 10 high-power fields, and vascular proliferation qualify for anaplastic oligodendroglioma, WHO grade III. Necrosis often accompanies vascular proliferation and mitotic activity, but is not required. $\mathbf{f}$ Gemistocytic astrocytoma appears as cells with abundant, glassy, eosinophilic cytoplasm, and frequently is associated with perivascular inflammatory infiltrates. This astrocytoma subtype has a propensity toward rapid malignant progression

$[15,32]$. Gemistocytic astrocytoma is a variant characterized morphologically by at least $20 \%$ of the neoplastic cells showing abundant eosinophilic cytoplasm and short branching processes (Fig. 2f). These tumors may have a more aggressive course and frequently show copy number gain of chromosome arm 12p encompassing the cyclin D2 (CCND2) locus [33]. A few glioblastoma histologic subtypes are recognized as distinct entities by the $2016 \mathrm{WHO}$, such as epithelioid glioblastoma, giant cell glioblastoma, and gliosarcoma. Epithelioid glioblastoma is a new addition to the WHO 2016 [15]. These tumors mostly occur in younger patients, and about half show BRAF V600E mutations; the differential diagnosis with anaplastic pleomorphic xanthoastrocytoma can be challenging and the relationship between these entities is controversial [34, 35]. Other histologic subtypes are considered morphologic variants, such as small cell glioblastoma, and glioblastoma with primitive neuronal component. These entities and their characteristic histologic features have been reviewed elsewhere [31].

While some infiltrating gliomas readily declare themselves morphologically as astrocytoma or oligodendroglioma, many are ambiguous and appear either as a mixture of both cell types, or with a constellation of nuclear features that defies reproducible categorization. Before 2016, such "in-between" diffuse gliomas were categorized as "oligoastrocytoma" and assigned WHO grade II, or grade III (anaplastic) in the setting of significant mitotic activity, necrosis, and/or vascular proliferation. Oligoastrocytomas had an intermediate prognosis between histologically defined diffuse astrocytomas and oligodendrogliomas, but diagnostic reproducibility for 
this category or tumors was poor [36, 37]. The WHO 2016 eliminated oligoastrocytoma as a distinct entity, since nearly all histologically defined oligoastrocytomas can be re-categorized as oligodendroglioma or astrocytoma based on molecular features [38]. Similarly, nearly all anaplastic oligoastrocytomas and glioblastomas with oligodendroglial component (essentially, a WHO grade IV oligoastrocytoma) declare themselves by molecular criteria as either anaplastic oligodendroglioma, anaplastic astrocytoma, or glioblastoma [39]. There are reports of diffuse gliomas with distinct oligodendroglial and astrocytic components by morphologic and molecular criteria, but such cases are exceptionally rare, and these are not recognized as a distinct entity by current criteria $[40,41]$. The WHO recognizes that in some diffuse glioma cases where molecular testing cannot be done, a tumor may fall into the old oligoastrocytoma category. In such cases, the diagnosis of "oligoastrocytoma, NOS" can be made, though such cases should be rare. If molecular testing results do not fit with an existing molecular category, the diagnosis of "oligoastrocytoma, not elsewhere classified" may be applied [25].

\section{Molecular classification of lower grade infiltrating gliomas: IDH mutations and 1p/19q-codeletion}

In 2008, next-generation sequencing identified recurrent mutations in isocitrate dehydrogenase 1 (encoded by IDH1) in a subset of glioblastoma cases, mostly from younger patients with a history of prior lower-grade astrocytoma (clinically referred to as secondary glioblastoma) [9]. Subsequent studies showed that recurrent missense mutations involve $I D H 1$ at position arginine 132, and less commonly in the homologous arginine 172 of $I D H 2$. Overall, IDH1 R132 and IDH2 R172 alterations occur in about $80-90 \%$ of adult WHO grade II or III infiltrating astrocytomas, oligodendrogliomas, and secondary glioblastoma [42]. Normally, the isocitrate dehydrogenase proteins -- collectively referred to as IDH -- catalyze the oxidative decarboxylation of isocitrate to $\alpha$-ketoglutarate. The mutant forms of IDH acquire a neomorphic activity and instead convert isocitrate to D-2-hydroxyglutarate, a so-called "oncometabolite" that builds to a very high level in tumor cells and interferes with several cellular processes, ultimately resulting in changes to DNA and histone methylation patterns that alter gene expression by establishing a glioma $\mathrm{CpG}$ island methylator phenotype (G-CIMP) [43, 44]. The most common form of mutant IDH is a missense mutation in IDH1 converting arginine at position 132 to histidine (IDH1 R132H). This mutant protein is detectable by a sensitive and specific antibody $[45,46]$. Immunopositivity for IDH1 R132H is sufficient to classify a glioma as "IDH-mutant" [15]. If the immunostain is negative, sequencing of $I D H 1$ and $I D H 2$ can be performed to assess for less common $I D H 1$ mutations, or mutations in $I D H 2$. These non-canonical alterations are very rare in patients over 54, so sequencing is generally not necessary in older patients with glioblastoma histology and no history of a prior lower grade glioma [25, 47, 48]. If sequencing cannot be performed or the necessary assays have failed, tumors receive a histologic diagnosis and the NOS qualifier.

Oligodendrogliomas now have a strict molecular definition and must show an IDH alteration and evidence for deletion of both the short arm of chromosome 1 (1p) and the long arm of chromosome 19 (19q) for an integrated diagnosis. $1 \mathrm{p} / 19 \mathrm{q}$-codeletion results from a reciprocal translocation between chromosomes 1 and 19 -$\mathrm{t}(1 ; 19)(\mathrm{p} 10 ; \mathrm{q} 10)$-- with subsequent loss of one derivative chromosome, leaving an imbalance and loss of $1 \mathrm{p}$ and $19 \mathrm{q}[49,50]$. For many years, fluorescence in situ hybridization (FISH) has been used to define the $1 \mathrm{p} /$ $19 \mathrm{q}$-codeletion, using molecular probes that align at the distal ends of the chromosome 1 and 19 arms. For example, probes from Abbott Molecular are located at $1 \mathrm{p} 36.2 / 1 \mathrm{q} 25.2$ and $19 \mathrm{p} 13.2 / 19 \mathrm{q} 13.3$, and the ratio of $1 \mathrm{p}$ to $1 \mathrm{q}$ and $19 \mathrm{q}$ to $19 \mathrm{p}$ is calculated to determine deletion. Recently, evidence has amassed that FISH is insufficient to fully distinguish oligodendrogliomas from other brain tumors (usually glioblastoma) that harbor focal deletions of $1 \mathrm{p}$ and $19 \mathrm{q}$ and thus give false positive results on FISH analysis [51, 52]. For this reason, the WHO 2016 recommends molecular testing by a method that assess whole-arm chromosomal loss, such as molecular inversion probe array, single nucleotide polymorphism chromosomal microarrays (hereafter abbreviated SNP microarrays), or next-generation sequencing with copy number analysis [20]. Our molecular diagnostics group has come to a similar conclusion, and whenever possible our glioma testing is now performed by SNP microarrays, occasionally showing that results of " $1 \mathrm{p} / 19 \mathrm{q}$-codeletion" by FISH are due to focal terminal or interstitial deletions rather than whole arm losses (Fig. 3, a-b). Another advantage is that this platform assesses genome-wide copy number changes, and alterations such as EGFR amplification, focal deletions on 9p encompassing $C D K N 2 A / B$, and focal or whole chromosome gains/losses can be detected. For laboratories not currently offering microarray methods, it may be possible to include additional, more proximal FISH probes (i.e. closer to the centromere) to increase confidence in whole arm codeletion, although this approach would increase technical and scoring complexity and potentially increases cost to a similar level as microarray platforms.

\section{The role of telomere maintenance: ATRX, p53, and the TERT promoter}

Replicative senescence occurs as a consequence of cell division. Incomplete replication of the chromosome ends 


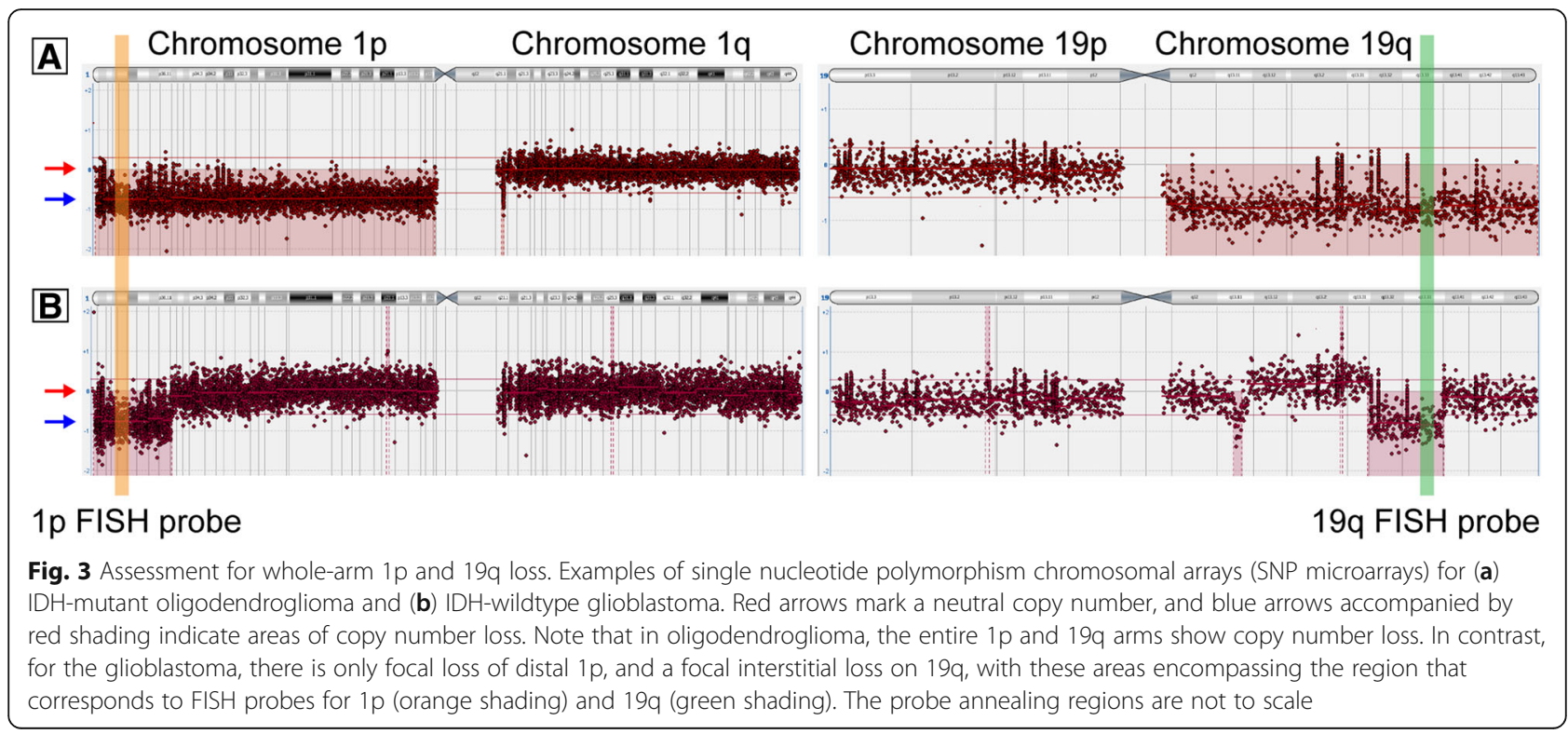

leads to progressive shortening of the telomeres, eventually triggering a DNA damage checkpoint signal resulting in cell cycle arrest [53]. Telomere length is maintained in stem/progenitor and germline cells by telomerase [53]. The telomerase holoenzyme consists of telomerase reverse transcriptase or TERT, a catalytic subunit encoded by the TERT gene on chromosome 5, dyskeratin, and template telomerase RNA component, which together act to extend telomeres through addition of a repetitive DNA sequence to the chromosome ends $[53,54]$. TERT expression is normally silenced in nearly all somatic cells, and a telomerase reactivation is an important factor in escape from replicative senescence, one of the hallmarks of cancer [55]. Recurrent, mutually exclusive point mutations in the TERT promoter were originally discovered in melanoma, and subsequently identified in other tumors, including at a high frequency in primary glioblastoma and oligodendroglioma [56-58]. Mechanistic studies later showed that TERT promoter mutations generate a cryptic binding site for an E26 transformation-specific (ETS) family transcription factor called GA-binding protein, alpha subunit (GABPA), leading to telomerase re-expression and subsequent telomere elongation [59]. Sequencing of the TERT promoter region is the only method to detect this alteration; in several studies of multiple cancer types, including gliomas, TERT immunohistochemistry does not correlate with promoter mutation status [56, 60-62]. Epigenetic mechanisms can also regulate TERT expression, and this may have prognosis in certain pediatric brain tumors, where TERT promoter mutations are rare [57, 63, 64]. Alternative mechanisms for telomere maintenance include TERT amplification and TERT promoter rearrangements $[65,66]$.
In contrast to oligodendrogliomas and IDH-wildtype glioblastoma, TERT promoter mutations are rare in IDH-mutant astrocytomas, which instead maintain telomere length through a mechanism involving mutations in the ATRX gene, usually accompanied by mutations in TP53. ATRX encodes $\alpha$-thalassemia/mental retardation syndrome X-linked, a protein that, along with death-domain associated protein or DAXX, is involved in maintaining chromatin structure at the telomeres [54]. ATRX alterations strongly correlate with a phenotype called alternative lengthening of telomeres (ALT), characterized by increased telomere homologous recombination and subsequent telomere elongation [67]. Within brain tumors, the ALT phenotype is frequent in IDH-mutant astrocytomas and histone $\mathrm{H} 3$ mutant gliomas.

Immunohistochemical markers provide insight into underlying molecular alterations and aid in glioma classification (Fig. 4) [17, 68]. Typical oligodendrogliomas are positive for IDH1 R132H unless they have a non-canonical IDH alteration, and generally show strong nuclear staining for ATRX and little to no nuclear reactivity for p53 (Fig. 4, a-d), except for anaplastic cases which can show a significant amount of p53 immunoreactivity. In astrocytomas, ATRX mutations are usually accompanied by loss of nuclear staining for ATRX protein, and TP53 mutations result in stabilization of the p53 protein leading to strong nuclear staining in a significant proportion of tumor nuclei (Fig. 4, e-h). ATRX immunoreactivity can be patchy because the stain is very sensitive to fixation and cautery, so before concluding that a diffuse glioma shows ATRX loss, internal positive control staining in the endothelial, inflammatory, and entrapped neuronal component in the same part of the 


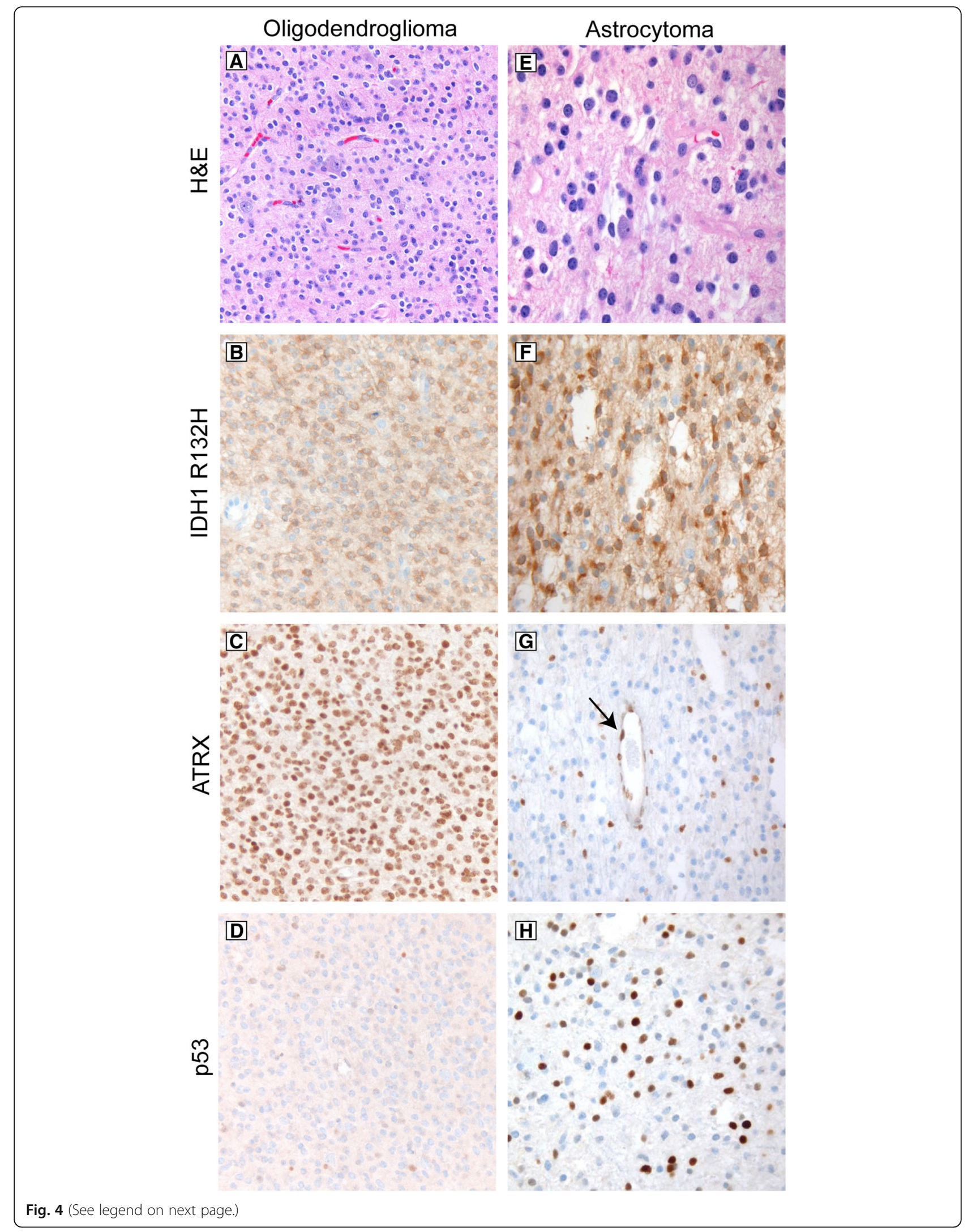


(See figure on previous page.)

Fig. 4 Immunohistochemical features in typical lower grade infiltrating gliomas. Oligodendrogliomas (a) are immunoreactive for IDH1 R132H (b) unless they have a less common non-canonical mutation, in which case sequencing of IDH1 and IDH2 is required; they lack ATRX mutations and therefore retain nuclear reactivity of ATRX protein in tumor cell nuclei (c), and p53 immunoreactivity is generally low (d), except in anaplastic cases. Astrocytomas with IDH mutation (e-f) and ATRX mutations almost always show loss of ATRX staining in tumor nuclei, while endothelial cells (black arrow) and entrapped normal cells are positive, providing an internal positive control (g). TP53 mutations are associated with increased staining in tumor cell nuclei (h)

sample should be identified (Fig. 4, g). Also, not all ATRX mutations are associated with loss of nuclear staining, so in a tumor with classic astrocytoma histology and a confirmed IDH1 or IDH2 mutation, positive nuclear staining for ATRX does not exclude the diagnosis of astrocytoma.

Immunohistochemistry for p53 bears some caveats. Most TP53 mutations are missense mutations that result in nuclear accumulation of the protein [69]. However, this immunostaining pattern is neither sensitive nor specific for a TP53 mutation and the result must be interpreted in context with morphology and other immunohistochemical and molecular findings. In one series of 157 diffuse gliomas, a cutoff of strong nuclear p53 reactivity in $>10 \%$ of tumor nuclei had a positive predictive value of $94.5 \%$ and negative predictive value of $86.3 \%$ for predicting TP53 mutation status [69]. Within brain tumors, TP53 mutations can be seen in $I D H$-wildtype glioblastomas, occasional circumscribed gliomas, embryonal tumors including medulloblastoma, other rare primary brain tumors such as choroid plexus neoplasms, and in metastatic tumors. Also, non-neoplastic conditions -- especially demyelinating processes -- can be associated with increased p53 nuclear staining, and this can be a particular pitfall in progressive multifocal leukoencephalopathy since that condition can cause significant astrocytic atypia [70]. Finally, absence of nuclear p53 staining does not exclude a TP53 mutation since truncating or splice site TP53 mutations are a mechanism for loss of p53 function, and this leads to loss of nuclear immunoreactivity due to decreased or absent protein expression [69]. Overall, with the appropriate tumor morphology of a diffuse or anaplastic astrocytoma, either ATRX loss or strong p53 staining in $>10 \%$ of tumor nuclei is sufficient to diagnose an astrocytoma of the appropriate grade without assessing $1 \mathrm{p} /$ 19q [28]. A combination of tumor morphology, IDH status, and the immunophenotype for IDH1-R132H and ATRX, aided by $\mathrm{p} 53$, is adequate to classify most diffusely infiltrating gliomas, with confirmatory assessment of $1 p / 19 q$ status in suspected oligodendrogliomas and sequencing of the IDH genes to detect suspected non-canonical alterations, and these modalities can be applied in a sequential or algorithmic approach [71, 72].

\section{Molecular characteristics of IDH-wildtype glioblastoma}

Most glioblastomas occur in adults, and 90\% are IDH-wildtype tumors that arise de novo, i.e. with a rapid clinical presentation and absent a precursor lower-grade lesion (primary glioblastoma). The remainder (about $10 \%)$ are mostly IDH-mutant tumors, typically arising from lower grade infiltrating astrocytomas, and thus clinically referred to as secondary glioblastoma. The molecular features of IDH-wildtype glioblastoma have been studied extensively, however the prognosis remains poor [1]. Amplification of double minute chromosomes of the epidermal growth factor receptor gene $(E G F R)$ is a frequent event in IDH-wildtype glioblastomas, while EGFR point mutations are comparatively rare. The EGFR gene also commonly incurs an intragenic deletion of exons 2-7 producing a constitutively active variant protein called EGFR-vIII, occurring in a quarter to half of glioblastoma. Expression of EGFR-vIII is almost always associated with amplification of the wild-type EGFR allele, but the clinical significance of either alteration is still unclear [73, 74]. Concomitant chromosome $7 p$ gain combined with chromosome 10q loss is the most frequent genetic alteration in glioblastoma, with almost half showing both alterations; a large fraction of EGFR amplification occurs on a background of chromosome 7 polysomy and chromosome 10 monosomy [75, 76].

The $\mathrm{O}^{6}$-methylguanine-DNA methyltransferase (MGMT) protein, encoded by the MGMT gene on chromosome 10, plays a role in repairing the DNA damage from alkylating agents, including temozolomide. Methylation of the MGMT promoter region is associated with silencing of gene expression, and occurs in about $40-50 \%$ of glioblastoma cases [15]. Mechanistic studies suggest that tumors with low MGMT are deficient in repairing temozolomide-induced DNA damage and therefore have higher chemosensitivity. In 2005, results from a randomized multicenter phase III clinical trial comparing radiotherapy to radiotherapy with concomitant temozolomide demonstrated MGMT promoter methylation as an independent favorable prognostic factor, and a predictive factor for survival benefit in patients treated with temozolomide and radiation therapy [77, 78]. Subsequent studies support MGMT promoter methylation as a predictive biomarker in glioblastoma, but the exact role in guiding clinical management is still being refined, partly due to challenges in testing methodology and establishing meaningful cutoffs for the assays [79]. 


\section{Molecular methods in IDH-wildtype glioblastoma}

FISH readily identifies EGFR amplification, but is being phased out in favor of chromosomal microarray, which can detect the amplification with better total copy number estimates and provides additional copy number information across the genome. The deleted region for EGFR-vIII is small, at only 13 kilobases, and therefore not amenable to FISH, and furthermore mosaicism complicates microarray detection and interpretation, particularly against the background of an amplified gene. Currently, of the genomic technologies readily available in the lab, next generation sequencing with high read depth is the most robust approach for detecting this deletion. Partial as well as whole chromosome gains and losses are readily observed using SNP microarray, which can circumvent some of the limitations of targeted FISH. For example, homozygous deletion of the DMBT1 gene on chromosome 10 (encoding Deleted In Malignant Brain Tumors 1 protein) has been reported in glioblastoma and medulloblastoma [80]. In tumors with diffuse astrocytoma histology, $D M B T 1$ loss may be predictive of a poor outcome, although IDH status was not assessed in that study so the status of DMBT1 as an independent predictor of outcome is unclear [81]. DMBT1 is located at chromosome 10q26.31, approximately 35 megabases distal to PTEN. Traditional FISH using a PTEN probe will not detect $D M B T 1$ deletion; SNP microarray will.

Copy number neutral loss of heterozygosity ( $\mathrm{LOH}$ ) is another common event in glioblastoma and can be detected using SNP chromosomal microarray [82]. LOH can uncover recessive mutations due to the transfer of one recessive allele onto both chromosome homologues via chromosomal crossover events, and $\mathrm{LOH}$ of $17 \mathrm{p}$, including the TP53 gene, is a frequent event [22]. Regional $\mathrm{LOH}$ may also prove useful in "gene discovery" efforts by identifying regions where a tumor suppressor or oncogene may reside. Additionally, SNP patterns may be more informative than oligonucleotides for large copy number changes only impacting a minority of cells, as the SNPs tend to be more sensitive to subtle changes associated with mosaicism. Finally, the use of both oligonucleotides and SNP microarrays has the advantage of one modality confirming the other, since the hybridizations are independent. Without micro-dissection in samples with a small tumor fraction (e.g. $<20 \%$ ), it becomes difficult to detect small changes (e.g. $<20 \mathrm{Mb}$ ) on microarray and FISH is a more sensitive technique. Thus, expert pathology review is critical for microarray studies, just as it has historically been for FISH.

Molecular methods for MGMT promoter methylation testing include simple or quantitative methylation-sensitive polymerase chain reaction, pyrosequencing, multiplex ligation-dependent probe amplification, and analysis by DNA methylation array. The relative costs and advantages/ disadvantages of each platform were recently reviewed [79]. Our institution uses pyrosequencing, but other methods could be chosen depending on specimen volume, testing costs, and other laboratory/technical factors.

\section{Histone H3 mutant gliomas}

Histones are a family of proteins that form a protein/ DNA complex to maintain chromatin structure and regulate gene expression, which can be mediated by post-translational histone modifications including methylation and acetylation. Histone octamers comprise two each of $\mathrm{H} 2 \mathrm{~A}, \mathrm{H} 2 \mathrm{~B}, \mathrm{H} 3$, and $\mathrm{H} 4$ proteins, and these are encoded by different genes. The histone $\mathrm{H} 3.3$ variant is expressed throughout the cell cycle and is encoded by $H 3 F 3 A$ and $H 3 F 3 B$, while the histone $\mathrm{H} 3.1$ variants are highly expressed during DNA replication and is encoded by several genes including HIST1H3B and HIST1H3C. Two categories of histone mutations have been identified in brain tumors. Sequencing studies of pediatric diffuse intrinsic pontine gliomas and non-brainstem glioblastomas identified recurrent mutations causing a lysine to methionine substitution at position 27 -- hereafter $\mathrm{K} 27 \mathrm{M}$-- in $H 3 F 3 A$ and $H I S T 1 H 3 B / C$, which are hereafter grouped as $\mathrm{H} 3$ to encompass $\mathrm{H} 3.3$ and H3.1 $[83,84]$. Very rare alternative alterations include a lysine to isoleucine alteration in $H 3 F 3 A$, and a $\mathrm{K} 27 \mathrm{M}$ alteration in histone $\mathrm{H} 2$ variant encoded by HIST2H3C [85]. The H3 K27M mutant protein has a dominant negative effect on the enhancer of zest 2 (EZH2) methyltransferase protein, which is a component of the polycomb repressive complex normally responsible for H3 K27 trimethylation [86]. Since the methionine residue of $\mathrm{H} 3 \mathrm{~K} 27 \mathrm{M}$ cannot be methylated, and also inhibits EZH2, the result is global alterations histone methylation and consequent dysregulation of gene expression [86]. The H3 K27M alteration has since been identified in midline gliomas across a spectrum of ages and tumor locations, including the third ventricle, pineal region, cerebellum, and spinal cord [87]. The WHO 2016 recognizes H3 K27M-mutant diffuse gliomas as a distinct molecular entity, and importantly these tumors correspond to WHO grade IV regardless of their histologic grade. The integrated diagnosis for these tumors is "Diffuse midline glioma, H3 K27M-mutant, WHO grade IV". Using the ISN-Haarlem system, this integrated diagnosis can go along with a histologic classification of diffuse astrocytoma, anaplastic astrocytoma, or glioblastoma, illustrating the usefulness of the layered reporting system since under different molecular circumstances a diffuse or anaplastic astrocytoma is WHO grade II or III, respectively.

Three examples of histone $\mathrm{H} 3$ mutant glioma are presented in Fig. 5, with H3 K27M-mutant tumors of the midbrain and thalamus presented in panels A-D and 

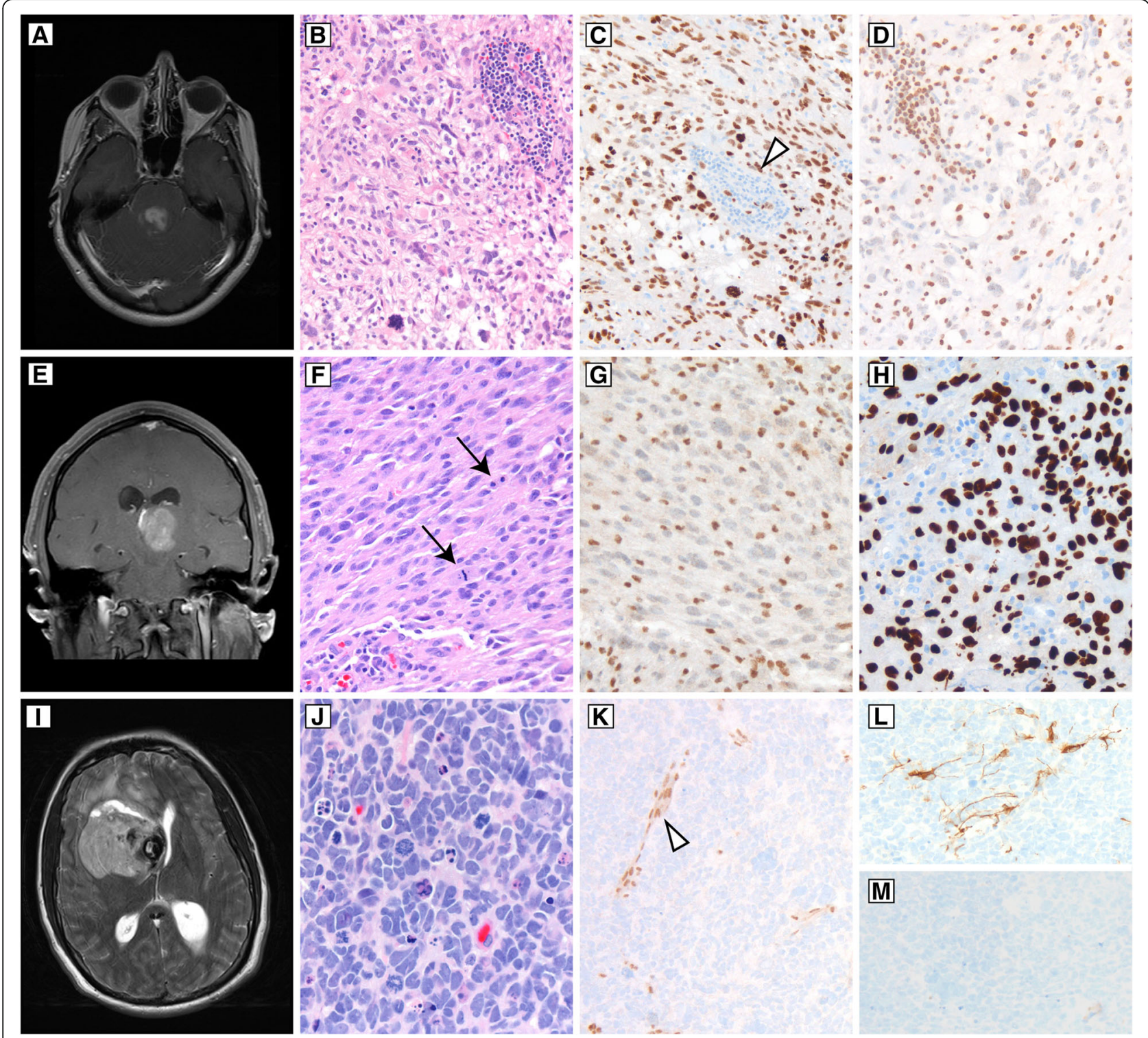

Fig. 5 Examples of histone H3 mutant gliomas. a Post-contrast T1-weighted imaging of an enhancing pontine mass occurring in a 33-year-old woman. Biopsy showed a markedly pleomorphic astrocytoma with frequent mitotic figures and prominent perivascular inflammation (b). Tumor cell nuclei were positive for histone H3 K27M mutant protein (c), while inflammatory cells were negative for this marker (white arrowhead). Immunohistochemistry for trimethylated histone $\mathrm{H3}$ is negative in tumor nuclei (d), and appropriately stains inflammatory cells. e Post-contrast T1-weighted imaging of a left thalamic tumor occurring in a 31-year-old man who presented with headaches. Sections showed an astrocytic neoplasm with mitotic activity (black arrows) (f). ATRX was absent in tumor cell nuclei, and stained a background population of macrophages and microglial cells (g). An immunostain for H3 K27M was positive in tumor nuclei (h), supporting the diagnosis of diffuse midline glioma, H3 K27M-mutant, WHO grade IV. (i) T2-weighted imaging of a right frontal cerebral hemispheric tumor in a 35-year-old man who presented with headaches. Sections showed a tumor with brisk mitotic activity, frequent apoptotic cells, high nuclear to cytoplasmic ratios, and nuclear molding (j). ATRX was negative in tumor cell nuclei (k), with preserved staining in the endothelial cells (white arrowhead). GFAP showed only focal staining (I), and OLIG2 was completely negative $(\mathbf{m})$. p53 (not pictured) was strongly positive. Sequencing of this tumor showed wildtype IDH1 and IDH2, and a further sequencing study revealed an H3F3A G34R mutation

$\mathrm{E}-\mathrm{H}$, respectively. The $\mathrm{H} 3 \mathrm{~K} 27 \mathrm{M}$ mutant protein is detectable by a highly sensitive and mutation-specific antibody (Fig. 5, c and h) [88]. However, since the K27M epitope is in a highly conserved region of the histone $\mathrm{H} 3$ family, the antibody does not distinguish mutations in H3F3A (H3.3-mutant) from HIST1H3B/C
(H3.1-mutant). This distinction may impact prognosis and eligibility for clinical trials, and in such cases sequencing of $H 3 F 3 A, H I S T 1 H 3 B / C$, and possibly HIST2H3C may be required [85, 88, 89]. Because the H3 K27M mutant protein suppresses histone $\mathrm{H} 3$ trimethylation by mechanisms described above, expression of $\mathrm{H} 3$ 
$\mathrm{K} 27 \mathrm{M}$ is associated with global reduction of histone $\mathrm{H} 3$ lysine position 27 trimethylation (H3 K27me3). This change is also detectable by immunohistochemistry (Fig. $5 \mathrm{~d}$ ), which reveals global loss of nuclear H3 K27me3 reactivity in tumor cells, however this is less specific than expression of the mutant protein, with the most common pitfall being focal loss of H3 K27me3 in atypical teratoid/rhabdoid tumor [88]. Importantly, global loss of histone $\mathrm{H} 3 \mathrm{~K} 27 \mathrm{me} 3$ is also an emerging prognostic indicator for an aggressive subgroup of posterior fossa ependymomas [90, 91]. The underlying mechanism for reduced H3 K27me3 in some ependymomas is unclear, but it occurs without the $\mathrm{H} 3 \mathrm{~K} 27 \mathrm{M}$ mutations that define diffuse midline gliomas. In practice, most $\mathrm{H} 3$ K27M-mutant diffuse midline gliomas will show both positive nuclear staining for $\mathrm{H} 3 \mathrm{~K} 27 \mathrm{M}$ mutant protein, and a corresponding loss of nuclear staining for $\mathrm{H} 3$ K27me3. For the mutation-specific antibody, the stain should show strong nuclear staining in tumor nuclei, and normal cellular components such as endothelial cells, inflammatory cells, and entrapped neurons should be negative (Fig. 5, c and h). Granular cytoplasmic staining is considered nonspecific. For H3 K27me3, non-neoplastic cellular components should be nuclear positive, and the tumor nuclei should be negative (Fig. $5 d)$.

When the WHO 2016 was published, H3 K27M mutations were thought to be specific for diffuse midline gliomas. However, H3 K27M mutations have now been reported in midline circumscribed gliomas such as ganglioglioma, pilocytic astrocytoma, unspecified glioneuronal tumors, and ependymoma, and in some series the clinical behavior of these tumors is more aggressive than histologically comparable H3 K27-wildtype counterparts, although it is not as dismal as for diffuse gliomas [92-95]. Intriguingly, H3 K27M mutations can co-occur with BRAF V600E, a common driver alteration in a variety of circumscribed gliomas and glioneuronal tumors $[19,94]$. In practice, this means that evidence for a $\mathrm{H} 3 \mathrm{~K} 27 \mathrm{M}$ mutation does not necessarily define the integrated diagnosis of diffuse midline glioma, WHO grade IV. The histologic context still matters: tumors must be definitively midline, diffusely infiltrating gliomas with a $K 27 \mathrm{M}$ alteration to qualify for the integrated diagnosis [28]. Due to the data supporting a worse clinical outcome of these rare $\mathrm{H} 3$ K27M-mutant circumscribed gliomas, our practice is to evaluate any midline ganglioglioma or pilocytic astrocytoma for H3 K27M mutant protein expression since these are the most common circumscribed histologic entities where $\mathrm{H} 3 \mathrm{~K} 27 \mathrm{M}$ has been reported.

H3F3A mutations also occur at guanine position 34, substituting arginine (G34R) or valine (G34V - hereafter $\mathrm{G} 34 \mathrm{R} / \mathrm{V}$ ) in histone H3.3, and an example of this is provided in Fig. 5, panels I through M. Unlike H3 K27M, the H3.3 G34R/V alteration has not been identified in the histone $\mathrm{H} 3.1$ isoforms encoded by HIST1H3B/C. The H3.3 G34R/V mutant tumors typically occur in the cerebral hemispheres in adolescents and young adults, and in a series of 81 cases almost always show loss of ATRX by immunohistochemistry (95\%), as well as TP53 mutation (88\%) [96]. Since the H3.3 G34R/V alteration is mutually exclusive with IDH mutations, H3.3 G34R/V should be considered in a hemispheric diffuse glioma that shows loss of ATRX but is proven by sequencing to be $I D H 1$ and $I D H 2$ wildtype. About one third of the H3.3 G34R/V mutant tumors show primitive neuronal features, with monomorphic cells showing a high nuclear to cytoplasmic ratio and largely lacking vascular proliferation or necrosis, and regardless of their histologic pattern (i.e. primitive neuronal or astrocytic) GFAP and OLIG2 expression may be limited (Fig. 5, j, l, and m) [96, 97]. An H3.3 G34R/V mutant tumor should also be considered in the differential diagnosis of a central nervous system primitive neuronal tumor, even if the typical glial markers are negative or focal/patchy. Most H3.3 G34R/V mutant tumors are MGMT promoter hypermethylated, which may contribute to a slightly better outcome in this group of tumors [96]. Since the H3.3 G34R/V is not currently a separate integrated diagnostic entity, these cases would be reported as astrocytomas of the appropriate histologic grade along with the "not elsewhere classified" modifier, and the molecular result of H3F3A G34R/V status should be reported [25]. The more general designation of astrocytic glioma, IDH-wildtype is not applicable since proven H3 G34R/V-mutant diffuse gliomas have another disease-defining molecular alteration and distinct clinical features [29]. Mutation-specific antibodies to detect H3.3 G34R or G34V mutant protein are in use at a few academic centers.

\section{Diffuse astrocytic glioma with molecular features of glioblastoma, WHO grade IV}

The WHO 2016 classifies glioblastoma into IDH-mutant and IDH-wildtype categories. This distinction has clinical utility in that multiple studies showed significantly better outcomes in IDH-mutant glioblastoma [13, 98]. In either molecular category, a tumor must still meet the appropriate histologic criteria to be diagnosed as a morphologic glioblastoma - namely tumors must show predominantly astrocytic differentiation with an infiltrative growth pattern, and exhibit microvascular proliferation and/or necrosis. Histologic parameters do not reliably distinguish IDH-wildtype from IDH-mutant tumors, although one recent and intriguing study showed that microthrombi are predictive of IDH-wildtype status [31, 99]. Prior to and since the publication of the WHO 
2016, studies have shown that some infiltrating astrocytomas that do not meet histologic criteria for glioblastoma may clinically behave in a manner similar to histologically defined glioblastoma. In particular, IDH-wildtype infiltrating astrocytomas, which would be histologically classified as grade WHO grade II or III, in many cases have worse outcomes than IDH-mutant glioblastomas, which are histologically grade IV, creating a significant problem in the current grading criteria [98, 100, 101].

The c-IMPACT-NOW consortium recently addressed this diagnostic dilemma by recommending a set of molecular features that indicate aggressive behavior in histologically lower grade, IDH-wildtype diffuse astrocytic gliomas [29]. Per these recommendations, diffuse astrocytic gliomas qualify for this designation with evidence of one or more of the following molecular alterations: (1) EGFR amplification, (2) TERT promoter mutation, and (3) whole-chromosome 7 gain combined with whole chromosome 10 loss (hereafter $+7 /-10$ ). Patients with a proven IDH-wildtype, histologically grade II or III infiltrating astrocytoma and any of these alterations can be diagnosed with "Diffuse astrocytic glioma, IDH-wildtype, with molecular features of glioblastoma, WHO grade IV", reflecting that their tumors are likely to behave in a manner similar to IDH-wildtype glioblastoma. These alterations may be seen alone or in combination, and they have varying sensitivity and specificity as individual parameters or in combination. Stitchel et al recently described these alterations across a large cohort of brain tumors that were categorized by global DNA methylation profiling (discussed below) [102]. DNA methylation profiling is an exciting technology with great promise for brain tumor classification and identifying molecular subgroups within and between histopathologic entities, and the tools for this analysis are publicly accessible through the German Cancer Research Center web portal at www. molecularneuropathology.org [103, 104]. As a molecular signature of glioblastoma in that series, EGFR amplification is the most specific but least sensitive marker, with 99.8\% specificity and $36.0 \%$ sensitivity. In this context, EGFR amplification refers to high level copy number gains; low level copy number gains and immunohistochemical expression of EGFR are not sufficiently specific for this purpose [105]. The $+7 /-10$ chromosomal alteration is also relatively specific $(98.0 \%)$ and was more sensitive that EGFR amplification (59.4\%). Although partial arm deletions and various combinations of chromosomes 7 gain and 10 loss may be seen in glioblastoma, partial arm alterations are not considered sufficient by cIMPACT-NOW criteria at this time [29, 102]. Finally, TERT promoter mutations have a clear association with aggressive behavior in the setting of glioblastoma, and this is the most sensitive (66.7\%) but least specific
(89.4\%) parameter, largely since TERT promoter mutations can be seen in other gliomas or systemic cancers [56, 57]. Critically, since TERT promoter mutations occur in a vast majority of molecularly defined (i.e. IDH-mutant and $1 \mathrm{p} / 19 \mathrm{q}$-codeleted) oligodendrogliomas, assessment of $1 \mathrm{p} / 19 \mathrm{q}$ status and exclusion of an IDH mutation by sequencing is usually required before designating a molecular glioblastoma.

Another complicating factor is the presence of other genetic alterations which may preclude a molecular glioblastoma diagnosis because of their own prognostic implications. The presence of $\mathrm{H} 3 \mathrm{~K} 27 \mathrm{M}$ in an infiltrative astrocytoma is diagnostic of diffuse midline glioma, H3 K27-mutant, a separate molecularly defined entity. Similarly, the IDH-wildtype gliomas with histone H3.3 G34R/V mutation should be considered, especially in younger patients [96]. Emerging molecular entities such as diffuse astrocytomas with $M Y B / M Y B L$ fusion, FGFR1 or FGFR3 alterations, or BRAF alterations are associated with a more indolent clinical course, but do not yet have their own diagnostic categorization and would appear as IDH-wildtype [106]. Recently, through DNA methylation profiling of histologically defined anaplastic pilocytic astrocytoma, Reinhardt et al defined the entity of anaplastic astrocytoma with piloid features, a category of IDH-wildtype astrocytoma with piloid morphology and frequent MAPK pathway alterations, loss of $C D K N 2 A / B$, and loss of ATRX. These tumors have a better outcome than IDH-wildtype glioblastoma and are molecularly distinct from conventional glioblastoma groups, as defined by DNA methylation profiling [107]. Finally, there are diffuse astrocytic gliomas which lack IDH mutation, EGFR amplification, TERT promoter mutation, and the $+7 /-10$ chromosomal alteration. In the absence of an identifiable molecular driver, such cases may be diagnosed by histologic features and designated as "Not elsewhere classified".

In summary, for these three alterations, their presence alone is not sufficient for the molecular glioblastoma diagnosis and does so only with the histologic exclusion of other molecular-pathologic entities that may mimic diffuse astrocytomas, such as oligodendroglioma, other genetically-defined gliomas, or focal areas of infiltration in pleomorphic xanthoastrocytoma or pilocytic astrocytoma. The lack of absolute specificity for most of the alterations mentioned in this section, and the complexity of interpreting molecular findings in context of the histology, underscores the need for periodic updates to the molecular classification system. One possible approach to identifying these cases is presented in Fig. 6. Within the context of a diffuse astrocytic neoplasm, based on the tumor age and location, H3-mutant tumors (either H3 K27M or H3.3 G34R/V) can be considered, along with molecular features of glioblastoma which, if 


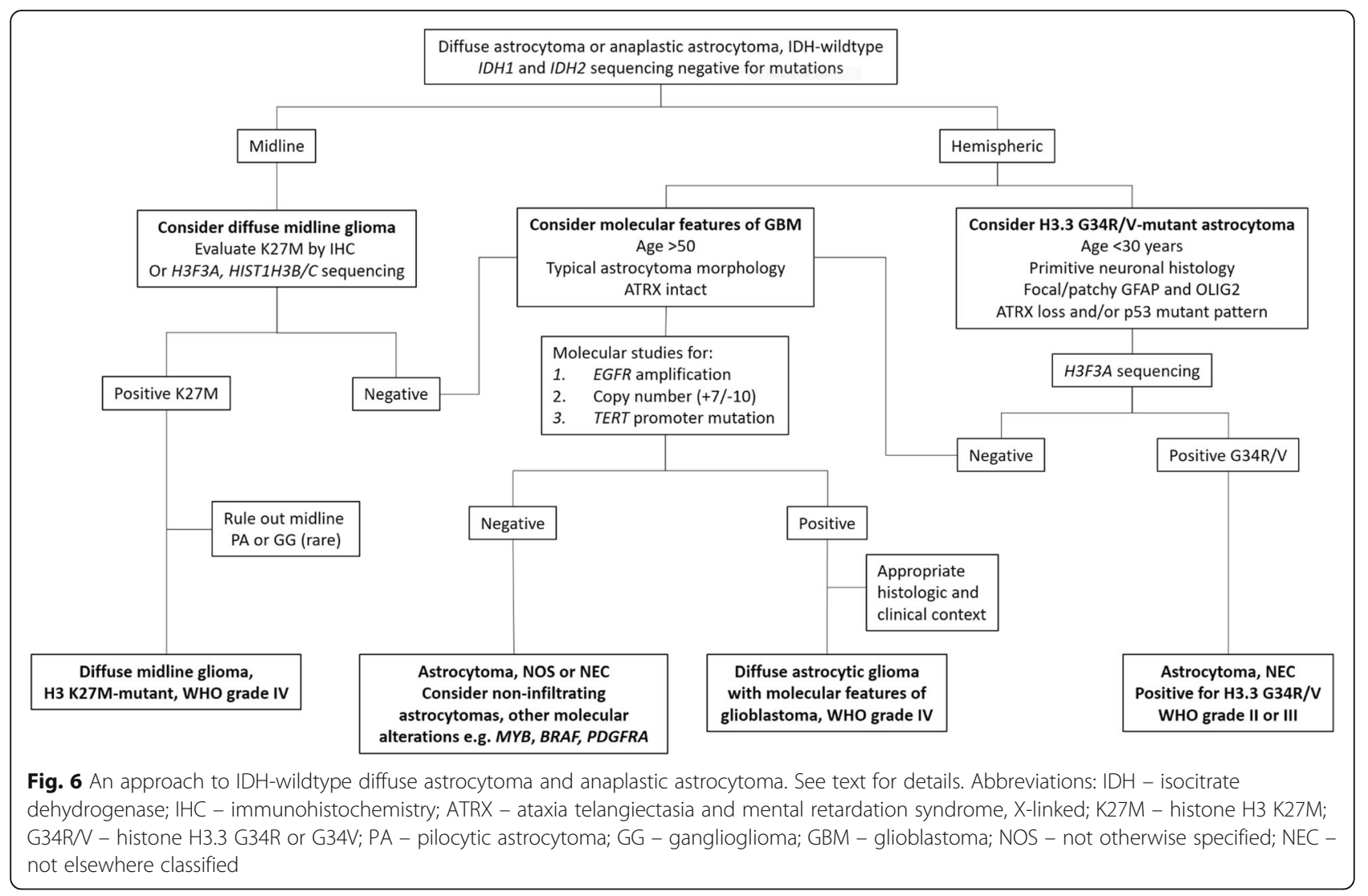

identified, should be taken in the proper clinical, radiographic, and histologic context. An integrated diagnosis and designation as WHO grade IV explicitly informs clinicians of the prognostic implications of the key molecular findings.

\section{Conclusions}

Brain tumor classification is increasingly rooted in the molecular drivers underlying different tumor entities. Currently, the absolute number of integrated diagnostic entities is low relative to the rapidly growing body of research on molecular analysis of brain tumors. However, incorporation of molecular features into the classification of diffuse gliomas means that due to their frequency, a majority of the primary intra-axial brain tumors now require some degree of molecular data for accurate and up-to-date classification. Additional molecularly defined entities and integrated diagnostic approaches are expected as the field evolves. As the number and scope of brain tumor-associated molecular alterations increases, academic medical centers are moving toward next-generation molecular diagnostics, allowing for detection of a broad spectrum of molecular alterations [104, 108-112]. Rich and useful data can still be obtained with careful morphologic analysis, judicious use of immunohistochemical stains, targeted sequencing, and fluorescence in situ hybridization, technologies which are accessible at most institutions and together can give adequate classification of most infiltrating gliomas. Pathologists should recognize that rare molecular entities exist, and that in some cases additional molecular testing may be indicated to detect clinically relevant alterations that are associated with aggressive behavior, such as molecular features of glioblastoma in histologically lower grade diffuse astrocytic gliomas. Molecular information now forms an essential part in neuropathology diagnosis, and the repertoire of molecular testing options will grow and evolve over time.

\section{Abbreviations}

ALT: Alternative lengthening of telomeres; ATRT: Atypical teratoid rhabdoid tumor; ATRX: a-thalassemia/mental retardation syndrome X-linked protein; CIMPACT-NOW: Consortium to Inform Molecular and Practical Approaches to CNS Tumor Taxonomy - Not Official WHO; CNS: Central nervous system; DAXX: Death-domain associated protein; EGFR: Epidermal growth factor receptor; ETMR: Embryonal tumor with multilayered rosettes; ETS: E26 transformation-specific; EZH2: Enhancer of zest 2; FISH: Fluorescence in situ hybridization; GABPA: GA-binding protein, alpha subunit; GBM: Glioblastoma; G-CIMP: Glioma CpG island methylator phenotype; GFAP: Glial fibrillary acidic protein; GG: Ganglioglioma; H3 K27M: Histone H3 lysine 27 to methionine mutant protein; $\mathrm{H} 3 \mathrm{~K} 27 \mathrm{me} 3$ : Lysine position 27 trimethylated histone $\mathrm{H3}$; H3.3 G34R/G34V: Histone H3.3 guanine 34 to arginine or valine mutant protein; IDH: Isocitrate dehydrogenase; IDH1 R132H: Isocitrate dehydrogenase 1 arginine 132 to histidine mutant protein; IHC: Immunohistochemistry; ISN: International Society of Neuropathologists; LOH: Loss of heterozygosity; MAPK: Mitogen-activated protein kinase; 
MGMT: $\mathrm{O}^{6}$-methylguanine-DNA methyltransferase; NEC: Not elsewhere classified; NOS: Not otherwise specified; OLIG2: Oligodendrocyte transcription factor 2; PA: Pilocytic astrocytoma; SNP: Single nucleotide polymorphism; TERT: Telomerase reverse transcriptase protein; WHO 2016: WHO Classification of Tumours of the Central Nervous System, Revised 4th Edition; WHO: World Health Organization

\section{Acknowledgements}

We thank Andrew Hamilton for assistance with the literature search, and Dr. Marjorie Grafe comments and critical review of the manuscript.

\section{Funding}

Not applicable.

\section{Availability of data and materials}

Not applicable.

\section{Authors' contributions}

MDW, AMH, and SRM researched literature for the article, discussed and selected the content, wrote portions of the manuscript, and edited the final version of the manuscript. MDW performed literature searches, finalized selection of the topics, compiled and reviewed the references, identified and photographed exemplary cases, and created the Figures. All authors read and approved the final manuscript.

\section{Ethics approval and consent to participate}

Clinical data and photomicrographs are gathered under a Neurosurgery/ Neuropathology Data Repository, which has been approved by the Oregon Health \& Science University Institutional Review Board, with a waiver of patient consent (STUDY0018895).

\section{Consent for publication}

Not applicable.

\section{Competing interests}

The authors declare that they have no competing interests.

\section{Publisher's Note}

Springer Nature remains neutral with regard to jurisdictional claims in published maps and institutional affiliations.

\section{Author details}

'OHSU Department of Pathology, Division of Anatomic Pathology, Section of Neuropathology, Oregon Health \& Science University, 3181 SW Sam Jackson Park Road, L-113, Portland, OR 97213, USA. ${ }^{2}$ Knight Diagnostic Laboratories and Department of Molecular and Medical Genetics, Oregon Health \& Science University, Portland, OR 97239, USA.

Received: 20 November 2018 Accepted: 12 March 2019 Published online: 09 April 2019

\section{References}

1. Ostrom QT, Gittleman H, Truitt G, Boscia A, Kruchko C, Barnholtz-Sloan JS. CBTRUS Statistical Report: Primary Brain and Other Central Nervous System Tumors Diagnosed in the United States in 2011-2015. Neuro-Oncology. 2018;20(suppl_4):iv1-iv86.

2. Phillips J, Tihan T, Fuller G. Practical molecular pathology and histopathology of embryonal tumors. Surg Pathol Clin. 2015;8(1):73-88.

3. Stone TJ, Rowell R, Jayasekera BAP, Cunningham MO, Jacques TS. Review: molecular characteristics of long-term epilepsy-associated tumours (LEATs) and mechanisms for tumour-related epilepsy (TRE). Neuropathol Appl Neurobiol. 2018:44(1):56-69.

4. Blumcke I, Aronica E, Becker A, Capper D, Coras R, Honavar M, et al. Lowgrade epilepsy-associated neuroepithelial tumours - the 2016 WHO classification. Nat Rev Neurol. 2016;12(12):732-40.

5. Bailey OT. Genesis of the Percival Bailey-Cushing classification of gliomas. Pediatr Neurosci. 1985;12(4-5):261-5.

6. Ferguson S, Lesniak MS. Percival Bailey and the classification of brain tumors. Neurosurg Focus. 2005;18(4):e7.
7. Bailey $\mathrm{P}$, Cushing $\mathrm{H}$. A classification of the tumors of the glioma group on a Histogenetic basis with a correlated study of prognosis. Philadelphia: Lippincott; 1926.

8. Cairncross JG, Ueki K, Zlatescu MC, Lisle DK, Finkelstein DM, Hammond RR et al. Specific genetic predictors of chemotherapeutic response and survival in patients with anaplastic oligodendrogliomas. J Natl Cancer Inst. 1998. 90(19):1473-9.

9. Parsons DW, Jones S, Zhang X, Lin JC, Leary RJ, Angenendt P, et al. An integrated genomic analysis of human glioblastoma multiforme. Science. 2008;321(5897):1807-12

10. Lapointe S, Perry A, Butowski NA. Primary brain tumours in adults. Lancet. 2018;392(10145):432-46.

11. Eckel-Passow JE, Lachance DH, Molinaro AM, Walsh KM, Decker PA, Sicotte $\mathrm{H}$, et al. Glioma groups based on 1p/19q, IDH, and TERT promoter mutations in tumors. N Engl J Med. 2015;372(26):2499-508.

12. Cancer Genome Atlas Research N, Brat DJ, Verhaak RG, Aldape KD, Yung WK, Salama SR, et al. Comprehensive, integrative genomic analysis of diffuse lower-grade gliomas. N Engl J Med. 2015;372(26):2481-98.

13. Weller M, Weber RG, Willscher E, Riehmer V, Hentschel B, Kreuz M, et al. Molecular classification of diffuse cerebral WHO grade I//II gliomas using genome- and transcriptome-wide profiling improves stratification of prognostically distinct patient groups. Acta Neuropathol. 2015;129(5):679-93.

14. Louis DN, Perry A, Reifenberger G, von Deimling A, Figarella-Branger D, Cavenee WK, et al. The 2016 World Health Organization classification of tumors of the central nervous system: a summary. Acta Neuropathol. 2016; 131(6):803-20.

15. Louis DN, Ohgaki H, Wiestler OD, Cavanee WK. World Health Organization histological classification of Tumours of the central nervous system. France: International Agency for Research on Cancer; 2016.

16. Wesseling P, Capper D. WHO 2016 classification of gliomas. Neuropatho Appl Neurobiol. 2018;44(2):139-50.

17. Ferris SP, Hofmann JW, Solomon DA, Perry A. Characterization of gliomas: from morphology to molecules. Virchows Arch. 2017;471(2):257-69.

18. Reifenberger $\mathrm{G}$, Wirsching $\mathrm{HG}$, Knobbe-Thomsen $\mathrm{CB}$, Weller M. Advances in the molecular genetics of gliomas - implications for classification and therapy. Nat Rev Clin Oncol. 2017:14(7):434-52.

19. Chiang JC, Ellison DW. Molecular pathology of paediatric central nervous system tumours. J Pathol. 2017:241(2):159-72.

20. Velazquez Vega JE, Brat DJ. Incorporating advances in molecular pathology into brain tumor diagnostics. Adv Anat Pathol. 2018;25(3):143-71.

21. Sahm F, Reuss DE, Giannini C. WHO 2016 classification: changes and advancements in the diagnosis of miscellaneous primary CNS tumours. Neuropathol Appl Neurobiol. 2018;44(2):163-71.

22. Masui K, Mischel PS, Reifenberger G. Molecular classification of gliomas. Handb Clin Neurol. 2016:134:97-120.

23. Lopes MBS. The 2017 World Health Organization classification of tumors of the pituitary gland: a summary. Acta Neuropathol. 2017;134(4):521-35.

24. Louis DN, Perry A, Burger P, Ellison DW, Reifenberger G, von Deimling A, et al. International society of neuropathology--Haarlem consensus guidelines for nervous system tumor classification and grading. Brain Pathol. 2014; 24(5):429-35.

25. Louis DN, Wesseling P, Paulus W, Giannini C, Batchelor TT, Cairncross JG, et al. CIMPACT-NOW update 1: not otherwise specified (NOS) and not elsewhere classified (NEC). Acta Neuropathol. 2018;135(3):481-4.

26. Louis DN, Aldape K, Brat DJ, Capper D, Ellison DW, Hawkins C, et al. Announcing CIMPACT-NOW: the consortium to inform molecular and practical approaches to CNS tumor taxonomy. Acta Neuropathol. 2017;133(1):1-3.

27. Louis DN, Aldape K, Brat DJ, Capper D, Ellison DW, Hawkins C, et al. CIMPACT-NOW (the consortium to inform molecular and practical approaches to CNS tumor taxonomy): a new initiative in advancing nervous system tumor classification. Brain Pathol. 2017;27(6):851-2.

28. Louis DN, Giannini C, Capper D, Paulus W, Figarella-Branger D, Lopes MB, et al. CIMPACT-NOW update 2: diagnostic clarifications for diffuse midline glioma, H3 K27M-mutant and diffuse astrocytoma/anaplastic astrocytoma, IDH-mutant. Acta Neuropathol. 2018;135(4):639-42.

29. Brat DJ, Aldape $K$, Colman H, Holland EC, Louis DN, Jenkins RB, et al. CIMPACT-NOW update 3: recommended diagnostic criteria for "diffuse astrocytic glioma, IDH-wildtype, with molecular features of glioblastoma, WHO grade IV". Acta Neuropathol. 2018;136(5):805-10.

30. Practical Surgical Neuropathology. A Diagnostic Approach. 2nd ed. Philadelphia: Elsevier; 2018. 
31. Perry A, Wesseling P. Histologic classification of gliomas. Handb Clin Neurol. 2016;134:71-95.

32. Wesseling $\mathrm{P}$, van den Bent M, Perry A. Oligodendroglioma: pathology, molecular mechanisms and markers. Acta Neuropathol. 2015;129(6):809-27.

33. Sahm F, Korshunov A, Schrimpf D, Stichel D, Jones DT, Capper D, et al. Gain of $12 p$ encompassing CCND2 is associated with gemistocytic histology in IDH mutant astrocytomas. Acta Neuropathol. 2017;133(2):325-7.

34. Alexandrescu S, Korshunov A, Lai SH, Dabiri S, Patil S, Li R, et al. Epithelioid glioblastomas and anaplastic epithelioid pleomorphic Xanthoastrocytomas-same entity or first cousins? Brain Pathol. 2016;26(2):215-23.

35. Korshunov A, Chavez L, Sharma T, Ryzhova M, Schrimpf D, Stichel D, et al. Epithelioid glioblastomas stratify into established diagnostic subsets upon integrated molecular analysis. Brain Pathol. 2018;28(5):656-62.

36. van den Bent MJ. Interobserver variation of the histopathological diagnosis in clinical trials on glioma: a clinician's perspective. Acta Neuropathol. 2010; 120(3):297-304.

37. Giannini C, Scheithauer BW, Weaver AL, Burger PC, Kros JM, Mork S, et al. Oligodendrogliomas: reproducibility and prognostic value of histologic diagnosis and grading. J Neuropathol Exp Neurol. 2001;60(3):248-62.

38. Sahm F, Reuss D, Koelsche C, Capper D, Schittenhelm J, Heim S, et al. Farewell to oligoastrocytoma: in situ molecular genetics favor classification as either oligodendroglioma or astrocytoma. Acta Neuropathol. 2014;128(4):551-9.

39. Hinrichs BH, Newman S, Appin CL, Dunn W, Cooper L, Pauly R, et al. Farewell to GBM-O: genomic and transcriptomic profiling of glioblastoma with oligodendroglioma component reveals distinct molecular subgroups. Acta Neuropathol Commun. 2016;4:4.

40. Huse JT, Diamond EL, Wang L, Rosenblum MK. Mixed glioma with molecular features of composite oligodendroglioma and astrocytoma: a true "oligoastrocytoma"? Acta Neuropathol. 2015;129(1):151-3.

41. Wilcox P, Li CC, Lee M, Shivalingam B, Brennan J, Suter CM, et al. Oligoastrocytomas: throwing the baby out with the bathwater? Acta Neuropathol. 2015;129(1):147-9.

42. Yan H, Parsons DW, Jin G, McLendon R, Rasheed BA, Yuan W, et al. IDH1 and IDH2 mutations in gliomas. N Engl J Med. 2009;360(8):765-73.

43. Losman JA, Kaelin WG Jr. What a difference a hydroxyl makes: mutant IDH, (R)-2-hydroxyglutarate, and cancer. Genes Dev. 2013;27(8):836-52.

44. Duncan CG, Barwick BG, Jin G, Rago C, Kapoor-Vazirani P, Powell DR, et al. A heterozygous IDH1R132H/WT mutation induces genome-wide alterations in DNA methylation. Genome Res. 2012;22(12):2339-55.

45. Capper D, Sahm F, Hartmann C, Meyermann R, von Deimling A, Schittenhelm J. Application of mutant IDH1 antibody to differentiate diffuse glioma from nonneoplastic central nervous system lesions and therapyinduced changes. Am J Surg Pathol. 2010;34(8):1199-204.

46. Capper D, Zentgraf H, Balss J, Hartmann C, von Deimling A. Monoclonal antibody specific for IDH1 R132H mutation. Acta Neuropathol. 2009;118(5):599-601.

47. Chen L, Voronovich Z, Clark K, Hands I, Mannas J, Walsh M, et al. Predicting the likelihood of an isocitrate dehydrogenase 1 or 2 mutation in diagnoses of infiltrative glioma. Neuro-Oncology. 2014;16(11):1478-83.

48. DeWitt JC, Jordan JT, Frosch MP, Samore WR, lafrate AJ, Louis DN, et al. Cost-effectiveness of IDH testing in diffuse gliomas according to the 2016 WHO classification of tumors of the central nervous system recommendations. Neuro-Oncology. 2017;19(12):1640-50.

49. Griffin CA, Burger $P$, Morsberger $L$, Yonescu R, Swierczynski S, Weingart JD, et al. Identification of $\operatorname{der}(1 ; 19)(q 10 ; p 10)$ in five oligodendrogliomas suggests mechanism of concurrent $1 \mathrm{p}$ and $19 \mathrm{q}$ loss. J Neuropathol Exp Neurol. 2006;65(10):988-94

50. Jenkins RB, Blair H, Ballman KV, Giannini C, Arusell RM, Law M, et al. A t(1: 19)(q10;p10) mediates the combined deletions of $1 p$ and $19 q$ and predicts a better prognosis of patients with oligodendroglioma. Cancer Res. 2006; 66(20):9852-61.

51. Ballester LY, Huse JT, Tang G, Fuller GN. Molecular classification of adult diffuse gliomas: conflicting IDH1/IDH2, ATRX, and 1p/19q results. Hum Pathol. 2017:69:15-22.

52. Vogazianou AP, Chan R, Backlund LM, Pearson DM, Liu L, Langford CF, et al. Distinct patterns of $1 p$ and $19 q$ alterations identify subtypes of human gliomas that have different prognoses. Neuro-Oncology. 2010;12(7):664-78.

53. Xu L, Li S, Stohr BA. The role of telomere biology in cancer. Annu Rev Pathol. 2013;8:49-78

54. Lee J, Solomon DA, Tihan T. The role of histone modifications and telomere alterations in the pathogenesis of diffuse gliomas in adults and children. J Neuro-Oncol. 2017;132(1):1-11.
55. Hanahan D, Weinberg RA. The hallmarks of cancer. Cell. 2000;100(1):57-70.

56. Vinagre J, Almeida A, Populo H, Batista R, Lyra J, Pinto V, et al. Frequency of TERT promoter mutations in human cancers. Nat Commun. 2013;4:2185.

57. Koelsche C, Sahm F, Capper D, Reuss D, Sturm D, Jones DT, et al. Distribution of TERT promoter mutations in pediatric and adult tumors of the nervous system. Acta Neuropathol. 2013;126(6):907-15.

58. Killela PJ, Reitman ZJ, Jiao Y, Bettegowda C, Agrawal N, Diaz LA Jr, et al. TERT promoter mutations occur frequently in gliomas and a subset of tumors derived from cells with low rates of self-renewal. Proc Natl Acad Sci U S A. 2013;110(15):6021-6.

59. Bell RJ, Rube HT, Kreig A, Mancini A, Fouse SD, Nagarajan RP, et al. Cancer The transcription factor GABP selectively binds and activates the mutant TERT promoter in cancer. Science. 2015;348(6238):1036-9.

60. Paulsson JO, Olander A, Haglund F, Zedenius J, Juhlin CC. TERT immunohistochemistry is a poor predictor of TERT promoter mutations and gene expression in follicular thyroid carcinoma. Endocr Pathol. 2018;29(4): 380-3.

61. Hugdahl E, Kalvenes MB, Mannelqvist M, Ladstein RG, Akslen LA. Prognostic impact and concordance of TERT promoter mutation and protein expression in matched primary and metastatic cutaneous melanoma. Br J Cancer. 2018;118(1):98-105.

62. Masui K, Komori T, Kato Y, Masutomi K, Ichimura K, Ogasawara S, et al. Elevated TERT expression in TERT-wildtype adult diffuse gliomas: histological evaluation with a novel TERT-specific antibody. Biomed Res Int. 2018;2018: 7945845 .

63. Castelo-Branco P, Choufani S, Mack S, Gallagher D, Zhang C, Lipman T, et al. Methylation of the TERT promoter and risk stratification of childhood brain tumours: an integrative genomic and molecular study. The Lancet Oncology. 2013;14(6):534-42.

64. Ohba S, Mukherjee J, Johannessen TC, Mancini A, Chow TT, Wood M, et al. Mutant IDH1 expression drives TERT promoter reactivation as part of the cellular transformation process. Cancer Res. 2016;76(22):6680-9.

65. Valentijn $\sqcup$, Koster J, Zwijnenburg DA, Hasselt NE, van Sluis P, Volckmann R, et al. TERT rearrangements are frequent in neuroblastoma and identify aggressive tumors. Nat Genet. 2015;47(12):1411-4.

66. Piscuoglio S, Ng CK, Murray M, Burke KA, Edelweiss M, Geyer FC, et al. Massively parallel sequencing of phyllodes tumours of the breast reveals actionable mutations, and TERT promoter hotspot mutations and TERT gene amplification as likely drivers of progression. J Pathol. 2016;238(4):508-18.

67. Abedalthagafi M, Phillips JJ, Kim GE, Mueller S, Haas-Kogen DA, Marshall RE, et al. The alternative lengthening of telomere phenotype is significantly associated with loss of ATRX expression in high-grade pediatric and adult astrocytomas: a multi-institutional study of 214 astrocytomas. Mod Pathol. 2013;26(11):1425-32.

68. Tanboon J, Williams EA, Louis DN. The diagnostic use of Immunohistochemical surrogates for signature molecular genetic alterations in gliomas. J Neuropathol Exp Neurol. 2016;75(1):4-18.

69. Takami H, Yoshida A, Fukushima S, Arita H, Matsushita Y, Nakamura T, et al. Revisiting TP53 mutations and immunohistochemistry--a comparative study in 157 diffuse gliomas. Brain Pathol. 2015;25(3):256-65.

70. Dunbar E, Yachnis AT. Glioma diagnosis: immunohistochemistry and beyond. Adv Anat Pathol. 2010;17(3):187-201.

71. Reuss DE, Sahm F, Schrimpf D, Wiestler B, Capper D, Koelsche C, et al. ATRX and IDH1-R132H immunohistochemistry with subsequent copy number analysis and IDH sequencing as a basis for an "integrated" diagnostic approach for adult astrocytoma, oligodendroglioma and glioblastoma. Acta Neuropathol. 2015;129(1):133-46.

72. Mellai M, Annovazzi L, Senetta R, Dell'Aglio C, Mazzucco M, Cassoni P, et al. Diagnostic revision of 206 adult gliomas (including 40 oligoastrocytomas) based on ATRX, IDH1/2 and 1p/19q status. J NeuroOncol. 2017;131(2):213-22.

73. Shinojima N, Tada K, Shiraishi S, Kamiryo T, Kochi M, Nakamura H, et al. Prognostic value of epidermal growth factor receptor in patients with glioblastoma multiforme. Cancer Res. 2003;63(20):6962-70.

74. Gan HK, Cvrljevic AN, Johns TG. The epidermal growth factor receptor variant III (EGFRvIII): where wild things are altered. FEBS J. 2013;280(21): $5350-70$.

75. Koshiyama DB, Trevisan P, Graziadio C, Rosa RFM, Cunegatto B, Scholl J, et al. Frequency and clinical significance of chromosome 7 and 10 aneuploidies, amplification of the EGFR gene, deletion of PTEN and TP53 
genes, and $1 \mathrm{p} / 19 \mathrm{q}$ deficiency in a sample of adult patients diagnosed with glioblastoma from southern Brazil. J Neuro-Oncol. 2017;135(3):465-72.

76. Crespo I, Vital AL, Nieto AB, Rebelo O, Tao H, Lopes MC, et al. Detailed characterization of alterations of chromosomes 7, 9, and 10 in glioblastomas as assessed by single-nucleotide polymorphism arrays. J Mol Diagn. 2011; 13(6):634-47.

77. Hegi ME, Diserens AC, Gorlia T, Hamou MF, de Tribolet N, Weller M, et al. MGMT gene silencing and benefit from temozolomide in glioblastoma. N Engl J Med. 2005;352(10):997-1003.

78. Stupp R, Mason WP, van den Bent MJ, Weller M, Fisher B, Taphoorn MJ, et al. Radiotherapy plus concomitant and adjuvant temozolomide for glioblastoma. N Engl J Med. 2005;352(10):987-96.

79. Mansouri A, Hachem LD, Mansouri S, Nassiri F, Laperriere NJ, Xia D, et al. MGMT promoter methylation status testing to guide therapy for glioblastoma: refining the approach based on emerging evidence and current challenges. Neuro Oncol. 2018;21(2):167-78.

80. Mollenhauer J, Wiemann S, Scheurlen W, Korn B, Hayashi Y, Wilgenbus KK, et al. DMBT1, a new member of the SRCR superfamily, on chromosome 10q25.326.1 is deleted in malignant brain tumours. Nat Genet. 1997;17(1):32-9.

81. Motomura K, Mittelbronn M, Paulus W, Brokinkel B, Keyvani K, Sure U, et al. DMBT1 homozygous deletion in diffuse astrocytomas is associated with unfavorable clinical outcome. J Neuropathol Exp Neurol. 2012;71(8):702-7.

82. Kuga D, Mizoguchi M, Guan Y, Hata N, Yoshimoto K, Shono T, et al. Prevalence of copy-number neutral $\mathrm{LOH}$ in glioblastomas revealed by genomewide analysis of laser-microdissected tissues. Neuro-Oncology. 2008;10(6):995-1003.

83. Schwartzentruber J, Korshunov A, Liu XY, Jones DT, Pfaff E, Jacob K, et al. Driver mutations in histone $\mathrm{H} 3.3$ and chromatin remodelling genes in paediatric glioblastoma. Nature. 2012;482(7384):226-31.

84. Wu G, Broniscer A, McEachron TA, Lu C, Paugh BS, Becksfort J, et al. Somatic histone $\mathrm{H} 3$ alterations in pediatric diffuse intrinsic pontine gliomas and non-brainstem glioblastomas. Nat Genet. 2012;44(3):251-3.

85. Castel D, Philippe C, Calmon R, Le Dret L, Truffaux N, Boddaert N, et al. Histone H3F3A and HIST1H3B K27M mutations define two subgroups of diffuse intrinsic pontine gliomas with different prognosis and phenotypes. Acta Neuropathol. 2015;130(6):815-27.

86. Lewis PW, Muller MM, Koletsky MS, Cordero F, Lin S, Banaszynski LA, et al. Inhibition of PRC2 activity by a gain-of-function $\mathrm{H} 3$ mutation found in pediatric glioblastoma. Science. 2013;340(6134):857-61.

87. Solomon DA, Wood MD, Tihan T, Bollen AW, Gupta N, Phillips JJ, et al. Diffuse midline gliomas with histone H3-K27M mutation: a series of 47 cases assessing the Spectrum of morphologic variation and associated genetic alterations. Brain Pathol. 2016;26(5):569-80

88. Venneti S, Santi M, Felicella MM, Yarilin D, Phillips JJ, Sullivan LM, et al. A sensitive and specific histopathologic prognostic marker for H3F3A K27M mutant pediatric glioblastomas. Acta Neuropathol. 2014;128(5):743-53.

89. Castel D, Philippe C, Kergrohen T, Sill M, Merlevede J, Barret E, et al. Transcriptomic and epigenetic profiling of 'diffuse midline gliomas, H3 K27M-mutant' discriminate two subgroups based on the type of histone $\mathrm{H3}$ mutated and not supratentorial or infratentorial location. Acta Neuropathol Commun. 2018;6(1):117.

90. Pajtler KW, Witt H, Sill M, Jones DT, Hovestadt V, Kratochwil F, et al. Molecular classification of ependymal tumors across all CNS compartments, histopathological grades, and age groups. Cancer Cell. 2015;27(5):728-43.

91. Panwalkar P, Clark J, Ramaswamy V, Hawes D, Yang F, Dunham C, et al. Immunohistochemical analysis of $\mathrm{H} 3 \mathrm{~K} 27 \mathrm{me} 3$ demonstrates global reduction in group-a childhood posterior fossa ependymoma and is a powerful predictor of outcome. Acta Neuropathol. 2017;134(5):705-14.

92. Pratt D, Natarajan SK, Banda A, Giannini C, Vats P, Koschmann C, et al. Circumscribed/non-diffuse histology confers a better prognosis in H3K27Mmutant gliomas. Acta Neuropathol. 2018;135(2):299-301.

93. Kleinschmidt-DeMasters BK, Donson A, Foreman NK, Dorris K. H3 K27M mutation in Gangliogliomas can be associated with poor prognosis. Brain Pathol. 2017;27(6):846-50.

94. Pages M, Beccaria K, Boddaert N, Saffroy R, Besnard A, Castel D, et al. Cooccurrence of histone H3 K27M and BRAF V600E mutations in paediatric midline grade I ganglioglioma. Brain Pathol. 2018;28(1):103-11.

95. Joyon N, Tauziede-Espariat A, Alentorn A, Giry M, Castel D, Capelle L, et al. K27M mutation in H3F3A in ganglioglioma grade I with spontaneous malignant transformation extends the histopathological spectrum of the histone H3 oncogenic pathway. Neuropathol Appl Neurobiol. 2017:43(3): 271-6.
96. Korshunov A, Capper D, Reuss D, Schrimpf D, Ryzhova M, Hovestadt V, et al. Histologically distinct neuroepithelial tumors with histone 3 G34 mutation are molecularly similar and comprise a single nosologic entity. Acta Neuropathol. 2016;131(1):137-46.

97. Gessi M, Gielen GH, Hammes J, Dorner E, Muhlen AZ, Waha A, et al. H3.3 G34R mutations in pediatric primitive neuroectodermal tumors of central nervous system (CNS-PNET) and pediatric glioblastomas: possible diagnostic and therapeutic implications? J Neuro-Oncol. 2013;112(1):67-72.

98. Hartmann C, Hentschel B, Wick W, Capper D, Felsberg J, Simon M, et al. Patients with IDH1 wild type anaplastic astrocytomas exhibit worse prognosis than IDH1-mutated glioblastomas, and IDH1 mutation status accounts for the unfavorable prognostic effect of higher age: implications for classification of gliomas. Acta Neuropathol. 2010;120(6):707-18.

99. Unruh D, Schwarze SR, Khoury L, Thomas C, Wu M, Chen L, et al. Mutant IDH1 and thrombosis in gliomas. Acta Neuropathol. 2016;132(6):917-30.

100. Reuss DE, Kratz A, Sahm F, Capper D, Schrimpf D, Koelsche C, et al. Adult IDH wild type astrocytomas biologically and clinically resolve into other tumor entities. Acta Neuropathol. 2015;130(3):407-17.

101. Aibaidula A, Chan AK, Shi Z, Li Y, Zhang R, Yang R, et al. Adult IDH wild-type lower-grade gliomas should be further stratified. Neuro-Oncology. 2017; 19(10):1327-37.

102. Stichel D, Ebrahimi A, Reuss D, Schrimpf D, Ono T, Shirahata M, et al. Distribution of EGFR amplification, combined chromosome 7 gain and chromosome 10 loss, and TERT promoter mutation in brain tumors and their potential for the reclassification of IDHwt astrocytoma to glioblastoma. Acta Neuropathol. 2018;136(5):793-803.

103. Capper D, Jones DTW, Sill M, Hovestadt V, Schrimpf D, Sturm D, et al. DNA methylation-based classification of central nervous system tumours. Nature. 2018;555(7697):469-74

104. Capper D, Stichel D, Sahm F, Jones DTW, Schrimpf D, Sill M, et al. Practical implementation of DNA methylation and copy-number-based CNS tumor diagnostics: the Heidelberg experience. Acta Neuropathol. 2018;136(2):181-210.

105. Lee M, Kang SY, Suh YL. Genetic alterations of epidermal growth factor receptor in glioblastoma: the usefulness of immunohistochemistry. Appl Immunohistochem Mol Morphol. 2018. https://doi.org/10.1097/PAl. 0000000000000669.

106. Qaddoumi I, Orisme W, Wen J, Santiago T, Gupta K, Dalton JD, et al. Genetic alterations in uncommon low-grade neuroepithelial tumors: BRAF, FGFR1, and MYB mutations occur at high frequency and align with morphology. Acta Neuropathol. 2016;131(6):833-45.

107. Reinhardt A, Stichel D, Schrimpf D, Sahm F, Korshunov A, Reuss DE, et al. Anaplastic astrocytoma with piloid features, a novel molecular class of IDH wildtype glioma with recurrent MAPK pathway, CDKN2A/B and ATRX alterations. Acta Neuropathol. 2018;136(2):273-91.

108. Kline CN, Joseph NM, Grenert JP, van Ziffle J, Talevich E, Onodera C, et al. Targeted next-generation sequencing of pediatric neuro-oncology patients improves diagnosis, identifies pathogenic germline mutations, and directs targeted therapy. Neuro-Oncology. 2017;19(5):699-709.

109. Nikiforova MN, Wald Al, Melan MA, Roy S, Zhong S, Hamilton RL, et al. Targeted next-generation sequencing panel (GlioSeq) provides comprehensive genetic profiling of central nervous system tumors. NeuroOncology. 2016;18(3):379-87.

110. Sahm F, Schrimpf D, Jones DT, Meyer J, Kratz A, Reuss D, et al. Nextgeneration sequencing in routine brain tumor diagnostics enables an integrated diagnosis and identifies actionable targets. Acta Neuropathol. 2016;131(6):903-10.

111. Zacher A, Kaulich K, Stepanow S, Wolter M, Kohrer K, Felsberg J, et al. Molecular diagnostics of gliomas using next generation sequencing of a glioma-tailored gene panel. Brain Pathol. 2017;27(2):146-59.

112. Ramkissoon SH, Bi WL, Schumacher SE, Ramkissoon LA, Haidar S, Knoff D, et al. Clinical implementation of integrated whole-genome copy number and mutation profiling for glioblastoma. Neuro-Oncology. 2015;17(10):1344-55. 